reported a subjective improvement in symptoms and quality of life.

Conclusions Initiation of SV and dose optimisation in clinical practice represents a significant burden of additional work for heart failure teams. Dedicated, registrar-led outpatient clinics to monitor patients commenced on SV by heart failure specialists can successfully address this.

Prescribing within NICE TA388 guidelines in real world patients, there were similar drug tolerance and adverse event rates to those reported in PARADIGM-HF. However, the lower mean age within this particular population, who were carefully selected, may indicate that such findings are not representative of the entire heart failure population.

\section{A RETROSPECTIVE AUDIT OF MINERALOCORTICOID RECEPTOR ANTAGONIST (MRA) AND DEVICE THERAPY FOLLOWING MYOCARDIAL INFARCTION (MI) COMPLICATED BY LEFT VENTRICULAR (LV) SYSTOLIC DYSFUNCTION}

Jonathan Mailey, Daniel Nicholl*. Ulster Hospital, Dundonald

\subsection{6/heartjnl-2017-311726.3}

Introduction The EPHESUS trial demonstrated that eplerenone reduces mortality and hospitalisation from cardiac events in patients with symptomatic LV systolic dysfunction (LVSD) or diabetes following MI with LVEF 40\%. The SCD-HeFT and MADIT-II trials demonstrated a reduction in mortality with the use of primary preventative ICDs in heart failure with reduced ejection fraction (HF-rEF).

We conducted a retrospective audit of patients presenting with HF-rEF secondary to MI between $1 / 4 / 15$ and $31 / 3 / 16$. Our focus was on the initiation of MRA therapy, repeat assessment of LV function post MI and the use of device therapy if indicated.

Aims 1. Establish the proportion of eligible patients with a post MI LVEF $40 \%$ prescribed an MRA.

2. Identify whether patients with LVEF $35 \%$ are having LV imaging at 6-12 weeks and if severe LVSD persists whether device therapy is considered.

Methods Patients presenting with either NSTEMI or STEMI to The Ulster Hospital, Northern Ireland were identified using the hospitals MINAP database. Data regarding these patients was collected using electronic patient records and echo database. Each patient with an ejection fraction $40 \%$ with clinical heart failure or diabetes was considered eligible for MRA therapy provided not contraindicated by renal function or potassium.

In patients with LVEF 35\% we examined whether a repeat assessment of LV function with either echo or cardiac MRI was undertaken, when it was performed, and if appropriate whether ICD/CRT had been implanted. Patients were not considered eligible for a device if they had a pre-existing device, precluding co-morbidities, failed to attend echo or if they had died as an in-patient.

Results 350 patients presented to our institution with MI. 326 of these patients underwent IP assessment of LV function. $24 \%$ of the patients had LVEF $40 \%$ and $17 \%$ had LVEF $35 \%$. $14 \%$ of patients were considered appropriate candidates for MRA therapy, but only $47 \%$ of this cohort had been prescribed an MRA.
54 patients had LVEF 35\%, however only 27 were appropriate candidates for device consideration. $85 \%$ of appropriate candidates had a repeat assessment of LV function with a mean time to repeat imaging of 107 days. $17 \%$ of these patients had ongoing severe LVSD with the remainder having improved to $>35 \%$. Only $25 \%$ of those with ongoing severe LVSD had an ICD inserted, 25\% died prior to review and in $50 \%$ there was no documentation why device therapy had not been considered.

\begin{tabular}{ll} 
Abstract 3 Table 1 & \\
\hline Variable & $\mathbf{n}(\%)$ \\
\hline Patients with MI & $350(100 \%)$ \\
IP assessment LV function & $326(93 \%)$ \\
LVEF 40\% & $77(24 \%)$ \\
- Eligible for MRA & $47(14 \%)$ \\
MRA prescribed at discharge & $22(47 \%)^{*}$ \\
LVEF 35\% & $54(17 \%)$ \\
Eligible for device therapy & $27(50 \%)^{* *}$ \\
Not eligible for device: & $8(15 \%)^{* *}$ \\
- Died as IP & $3(6 \%)^{* *}$ \\
- Did not attend follow-up & $5(9 \%)^{* *}$ \\
- Device already in-situ & $11(20 \%)^{* *}$ \\
- Extensive co-morbidities & $23(85 \%)^{* * *}$ \\
- Repeat LV assessment & $4(15 \%)^{* * *}$ \\
- LVEF 35\% on repeat LV assessment & $1(25 \%)^{* * * *}$ \\
- ICD implanted & \\
\hline * = percentage of patients meeting eligibility criteria for MRA & \\
** = percentage of patients with LVEF 35\% & \\
*** percentage of patients with LVEF 35\% and appropriate for device therapy \\
*** $=$ percentage of patients eligible for primary preventative ICD after post discharge LV \\
assessment & \\
\hline
\end{tabular}

Conclusions This audit demonstrated that there is scope to improve our practice in the management of post MI patients with severe LVSD. Explanations for this may include staff education and resource pressures. The appropriate prescription of MRA therapy and implantation of ICDs have a big impact on mortality in this cohort. It is therefore of the upmost importance to address any potential barriers to compliance with guidelines in order to improve the quality of care that is delivered.

\section{$4 \quad$ IRON DEFICIENCY IN HEART FAILURE PATIENTS IN ENGLAND: INSIGHTS FROM ANALYSIS OF HOSPITAL EPISODE STATISTICS}

${ }^{1}$ James M Beattie*, ${ }^{2}$ Rani Khatib, ${ }^{3}$ Ceri Phillips, ${ }^{4}$ Simon G Williams. ${ }^{1}$ Heart of England NHS Foundation Trust; ${ }^{2}$ Leeds Teaching Hospitals NHS Trust and University of Leed; ${ }^{3}$ College of Human and Health Sciences, Swansea University; ${ }^{4}$ Wythenshawe Hospital

\subsection{6/heartjnl-2017-311726.4}

Introduction Iron deficiency (ID) has been shown to be present in about $50 \%$ of patients with heart failure (HF). Associated with a poor quality of life, impaired effort tolerance, and increased mortality, ID responds to appropriately provided iron therapy. In those admitted with HF, screening for ID is inconsistent, and the impact of this condition is uncertain. 
Methods For the period April 2013 to March 2016 (2013-16), Hospital Episode Statistics (HES) data from all NHS hospitals in England were evaluated for spells in which HF was coded as the primary diagnosis. This was based on the International Statistical Classification of Diseases and Related Health Problems (ICD-10) codes: I11.0 (hypertensive heart disease with [congestive] heart failure; I25.5 (ischaemic cardiomyopathy); I42.0 (dilated cardiomyopathy); I42.9 (cardiomyopathy, unspecified); I50.0 (congestive heart failure); I50.1 (left ventricular failure); and I50.9 (heart failure, unspecified). These records were then categorised according to those with or without a secondary diagnosis of ID or iron deficiency anaemia (IDA), based on ICD-10 codes E611 (latent ID), D500 (IDA secondary to blood loss [chronic]), D508 (other IDA), and D509 (IDA unspecified).

Results In 2013-16, there were 202444 hospital spells in England attributed to a primary diagnosis of HF. Of these, 28727 spells $(14.2 \%)$ had a secondary diagnosis of ID/IDA, and $173717(85.8 \%)$ did not. Spells with a secondary diagnosis of IDA/ID were more likely to be encountered in females $(p<0.0001)$ and older patients $(p<0.0001)$; were more likely to be unplanned $(95.9 \%$ vs $86.4 \%$ - difference 9.5\%: 95\% CIs: 9.2\%, 8\%); had a longer mean length of stay (16.5 vs 13.1 days - difference 3.4 days: 95\% CIs: 3.2, 3.6); and had a higher readmission rate within 30 days under the same ICD-10 code $(14.2 \%$ vs $12.1 \%$ - difference $2.1 \%$ : 95\%CIs: $1.8 \%, 2.6 \%)$. The total cost associated with all hospital admissions with a primary diagnosis of HF was $£ 553.3$ million, equivalent to $£ 2733$ per spell. HF hospital spells with a secondary diagnosis of ID/IDA were significantly more expensive than those without (cost difference: $£ 138$ per spell [95\% confidence interval $\{\mathrm{CI}\}: £ 98, £ 178]$ ). Unplanned spells with a secondary diagnosis of ID/IDA were even more expensive compared to those without ID/IDA (cost difference: $£ 217$ [95\% CI: £181, £253]).

Conclusions In this analysis of HES data from England, about $14 \%$ of hospital spells coded with a primary diagnosis of HF included ID/IDA in the secondary position. These spells were longer, more expensive, and more likely to lead to readmission. Although probably under recognised in those admitted with HF, ID/IDA appears to be a significant comorbidity associated with poorer outcomes across the health economy.

\section{THE RELATION BETWEEN LENGTH OF STAY, A\&E ATTENDANCE AND READMISSION FOR HEART FAILURE PATIENTS} ${ }^{1}$ Kate Honeyford*, ${ }^{2}$ Derek Bell, ${ }^{1}$ Paul Aylin, ${ }^{1}$ Alex Bottle. ${ }^{1}$ Dr Foster Unit, Imperial College;
${ }^{2}$ Imperial College

\subsection{6/heartjnl-2017-311726.5}

Introduction Many patients with heart failure (HF) have repeated hospitalisations, often unplanned. Hospitals are being penalised for what are considered to be preventable readmissions, but understanding of what drives high readmission rates is limited. Better understanding of the relationship between A and $\mathrm{E}$ attendance, the odds of admission during that attendance and overall hospital readmission rates will support hospitals to reduce overall readmissions and improve services for their HF patients.

Method Using admissions data for all acute hospitals in England (April 2010-March 2012), the index admission for each patient was defined as their first emergency admission with a primary diagnosis of $\mathrm{HF}$ for at least three years. A and $\mathrm{E}$ attendances, admissions and death within one year from index discharge were linked by patient. Hospital trusts were divided into quartiles based on their overall 30 day $\mathrm{HF}$ readmission rate. Logistic regression and ANOVA were used to identify any differences in $\mathrm{A}$ and $\mathrm{E}$ attendances, admissions and patient characteristics between hospital quartiles.

Results A total of 77801 patients had their first HF admission during the study period; 66177 (85\%) were discharged alive. Table 1 gives the first outcome within 30 days of that index discharge. Overall, 23\% of emergency readmissions were not through A and E. Patients who attended hospitals with readmission rates above the median were more likely to visit A and $\mathrm{E}$ than patients who were discharged from hospitals with lower readmission rates. Having arrived at A and E, the odds of admission was the same irrespective of hospital readmission rate, even after patient characteristics were taken into account.

The key difference between high- and low-readmitting hospitals appeared to be the length of stay of the index HF admission, with high-readmitting trusts having a higher proportion of patients discharged with no overnight stay these patients have the highest readmission rate.

\begin{tabular}{|c|c|c|c|c|}
\hline $\begin{array}{l}\text { Readmission Quartile } \\
\text { and number of patients }\end{array}$ & $\begin{array}{l}\text { Died without } \\
\text { readmission }\end{array}$ & $\begin{array}{l}\text { Readmitted - not } \\
\text { through } A \text { and } E\end{array}$ & $\begin{array}{l}\text { Visited } \\
A \text { and } E\end{array}$ & $\begin{array}{l}A \text { and } E \\
\text { admission } \\
\text { rate }\end{array}$ \\
\hline 1 & $359(2.6 \%)$ & $594(4.4 \%)$ & 2114 & $77.5 \%$ \\
\hline 13628 & & & $(15.5 \%)$ & (1638) \\
\hline 2 & $439(2.5 \%)$ & $1233(7.0 \%)$ & 2637 & $76.3 \%$ \\
\hline 17748 & & & $(14.9 \%)$ & (2013) \\
\hline 3 & $403(2.1 \%)$ & $869(4.5 \%)$ & 3807 & $76.5 \%$ \\
\hline 19167 & & & $(19.9 \%)$ & (2914) \\
\hline 4 & $377(2.4 \%)$ & $823(5.3 \%)$ & 3241 & $77.0 \%$ \\
\hline 15631 & & & $(20.7 \%)$ & (2496) \\
\hline \multirow[t]{2}{*}{ Totals } & $1578(2.4 \%)$ & $3519(5.3 \%)$ & 11799 & $76.8 \%$ \\
\hline & & & $(17.8 \%)$ & (9061) \\
\hline
\end{tabular}

Table 1 First outcome of heart failure patients within 30 days of their index discharge. Readmission quartile is based on the HF readmission rate of the index hospital.

Conclusions and Implications For patients with HF, high hospital-level readmission rates are partly driven by same-day index discharges, with their subsequent greater likelihood of attending $\mathrm{A}$ and $\mathrm{E}$, and more admissions via the GP or clinic, and not by admitting a higher proportion of $\mathrm{A}$ and $\mathrm{E}$ attenders. This suggests that patients who are same-day discharges are not inappropriate attenders but potentially prematurely sent home.

\section{\begin{tabular}{l|l}
6 & PROGNOSTIC VALUE OF MALNUTRITION SCREENING
\end{tabular} TOOLS IN PATIENTS WITH CHRONIC HEART FAILURE}

Shirley Sze*, Pierpaolo Pellicori, Alan Rigby, Syed Kazmi, Andrew Clark. Department of Cardiology, Castle Hill Hospital

10.1136/heartjnl-2017-311726.6

Background Malnutrition is a common clinical feature in patients with acute heart failure (AHF), but its prevalence and 